\title{
FORMULATION AND CHARACTERIZATION OF PAPAIN LOADED SOLID LIPID NANOPARTICLES AGAINST HUMAN COLORECTAL ADENOCARCINOMA CELL LINE
}

\author{
SURIYAKALA PERUMAL CHANDRAN*, KANNIKAPARAMESWARI NACHIMUTHU
}

Department of Biochemistry, Dr. N.G.P. Arts and Science College, Coimbatore, Tamil Nadu, India. Email: suriyakalabiochemsitry@gmail.com

Received: 11 May 2018, Revised and Accepted: 25 June 2018

\begin{abstract}
Objective: Colorectal cancer is one of the most commonly diagnosed cancer and also most common gastrointestinal malignancy with high prevalence rate in the younger population. Usually, cancer cells are surrounded by a fibrin coat which is resistant to fibrinolytic degradation. This fibrin coat is act as self-protective against natural killing mechanism. The main objective was to prepare papain-loaded solid lipid nanoparticles (P-SLN) by melt dispersion-ultrasonication method and investigated the cytotoxic efficacy against colorectal adenocarcinoma (human colorectal adenocarcinoma [HCT 15]) cells.
\end{abstract}

Methods: Optimized polymer ratio was characterized by differential scanning calorimetry, Fourier-transform infrared, X-ray diffraction, scanning electron microscopy, entrapment efficiency, particle size and zeta potential analysis, in vitro drug release, and in vitro cytotoxicity studies on HCT-15 colorectal adenocarcinoma cells.

Results: The results showed that the particle size, morphological character and zeta potential value of optimized batch P-SLN were 265 nm, spherical and $-26.5 \mathrm{Mv}$, respectively. The in vitro drug profile of P-SLN exhibited that it produced sustain drug release, and the cell viability of HCT-15 against P-SLN shown better efficacy than pure papain enzyme.

Conclusion: P-SLNs were successfully prepared and investigated the in vitro drug release and in vitro cell viability against HCT-15 cell line.

Keywords: Papain, Solid lipid nanoparticles, Cytotoxic potential, Proteolytic enzyme, In vitro drug release.

(C) 2018 The Authors. Published by Innovare Academic Sciences Pvt Ltd. This is an open access article under the CC BY license (http://creativecommons. org/licenses/by/4. 0/) DOI: http://dx.doi.org/10.22159/ajpcr.2018.v11i10.27258

\section{INTRODUCTION}

Colorectal cancer or bowl cancer is the second leading cause of mortality around the world, and more than 1.2 million patients are diagnosed with colorectal cancer every year, and more than 600,000 die from the disease [1]. Almost $25 \%$ of patients who present with colorectal cancer present with metastatic disease and thousands of patients receive treatment for metastatic CRC each year [2]. The approximate survival period for patients with metastatic CRC is around 20 months; however, most of the patients still die of their disease [3]. Since the launch, 5-fluorouracil (5-Fu) remains one of the most efficient and widely used drugs to treat colorectal cancer. On the other hand, inherent and acquired resistance is major obstacle to 5-Fu to achieve the effective clinical efficacy in CRC therapy. Therefore, it is essential to identify the mechanisms of resistance as a primary task to move toward to preventing or reversing chemoresistance in metastatic CRC patients [4]. The coating on the cancer cell is made of mucus and fibrin is one of the mechanism which responsible for chemoresistance. This fibrin (biofilm) protective coat prevents the white blood cells of the immune system from recognizing the cancer cell [5]. This protective fibrin layer may lead to the metastasis process because these cancer cells easily escape from immunosurveillance mechanism and also the chemotherapy not able to reach to the cancerous cells.

Proteolytic enzymes have the ability to prevent the formation of cancer metastases by dissolving the protective fibrin membrane which is encapsulated the cancer cells. Hence, a potent proteolytic enzyme has to propose to destroy the fibrin coat, exposing the cancer cell surface to the chemotherapy, and natural immune attack [6]. Papain is a proteolytic enzyme from the cysteine protease family which is the major constituent of papaya. It is believed that the proteolytic activity of papain may cleave the fibrin coat of the cancer cells without harming the normal cells. Since now, papain has not emerged any lipid-based drug delivery system to treat any type of cancer. Henceforth, we have proposed papain as a potent chemotherapeutic agent in treating colorectal cancer.

In spite of its wide pharmacological activity, it is unstable at room temperature and inactivated by acidic $\mathrm{pH}$ at the stomach [7]. Solid lipid nanoparticles (SLN) have been considered as a most promising drug delivery system to achieve better therapeutic outcome compared with conventional delivery. SLNs possess the numerous advantages than polymeric nanoparticles and liposomes [8]. SLNs are made of biodegradable and biocompatible lipidic carriers, which are principally solid state at room temperature [9]. Several investigations have revealed that SLNs have high drug loading capacity for both hydrophilic and lipophilic drugs [9], feasible for large-scale production [10], and long-term storage stability [11]. In addition, stealth SLNs with particle size $\leq 200 \mathrm{~mm}$ are infrequently subject to blood clearance by the reticuloendothelial system in the liver and spleen [12]. SLNs are flexible nanocarriers and are used for drug delivery in nearly all routes of administration, including ocular [13], parenteral [14], oral [15], and topical [16]. SLNs can be formulated to sustain the drug release profile and therefore decrease the necessity for the repeated administration and enhance the therapeutic value of the treatment [17]. The aim of this study was to load papain in SLNs by melt dispersion-ultrasonication technique and investigate the various formulation parameters, in vitro release studies, in vitro cytotoxicity, and stability studies. Papain-loaded SLN was prepared by melt dispersion-ultrasonication technique.

\section{MATERIALS AND METHODS}

Materials

Papain has received from Kotra Pharma, Malaysia, as gift sample; cetyl alcohol has purchased from Alpha Chemicals, India; 5-Fu has 
Table 1: Formulation P-SLN

\begin{tabular}{llllll}
\hline Batch & $\begin{array}{l}\text { Drug lipid } \\
\text { ratio }\end{array}$ & $\begin{array}{l}\text { Stirring Speed (rpm) } \\
\text { (at hot emulsion) }\end{array}$ & $\begin{array}{l}\text { Stirring time (min) } \\
\text { (at hot emulsion) }\end{array}$ & $\begin{array}{l}\text { Stirring } \\
\text { speed (rpm) } \\
\text { (at cold condition) }\end{array}$ & $\begin{array}{l}\text { Stirring time (min) } \\
\text { (at cold condition) }\end{array}$ \\
\hline B1 & $1: 1$ & 500 & 10 & 500 & 30 \\
B2 & $1: 2$ & 500 & 10 & 500 & 30 \\
B3 & $1: 3$ & 500 & 10 & 500 & 30 \\
B4 & $1: 4$ & 500 & 10 & 500 & 30 \\
B5 & $1: 5$ & 500 & 10 & 500 & 30 \\
B6 & $1: 5$ & 750 & 10 & 500 & 30 \\
\hline
\end{tabular}

P-SLN: Papain-loaded solid lipid nanoparticles

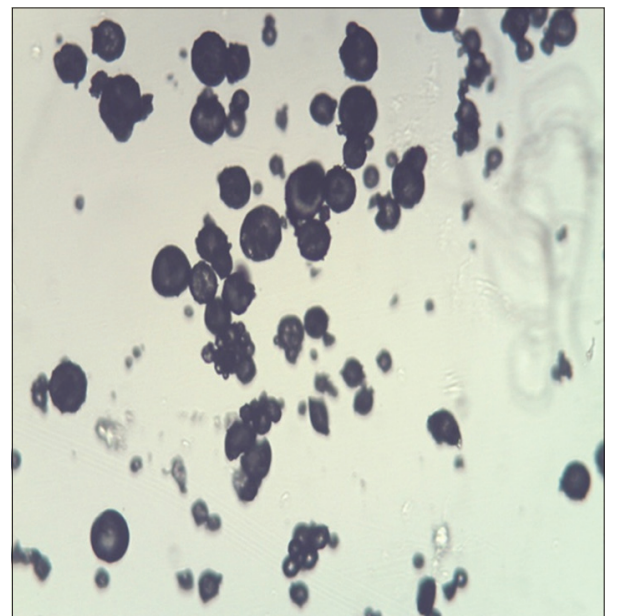

Fig. 1: Photomicroscopic image of papain-loaded solid lipid nanoparticles

purchased from Sisco Research Laboratories Pvt., Ltd, India; and all other chemicals and reagents were used as an analytical grade.

\section{Preparation of papain-loaded SLN (P-SLN)}

To attain better stability and sustain drug release profile, papain enzyme has encapsulated into SLN (P-SLN) by a modified melt dispersion method [18]. Cetyl alcohol is a class of triglycerides used as a carrier and acts as a non-ionic surfactant. Hence, P-SLN was prepared without the aid of any additional surfactant. The cetyl alcohol was melted and dispersed with papain enzyme, and this mixture was then instantaneously transferred to the hot aqueous phase (double distilled water). The hot coarse emulsion was homogenized for $10 \mathrm{~min}$ using mechanical stirrer (the speed variable mentioned in Table 1). The hot nanoemulsion $(\mathrm{o} / \mathrm{w})$ was immediately cool down to $5-10^{\circ} \mathrm{C}$ to obtain solid dispersion (P-SLNs). The P-SLNs were collected by centrifugation at 10,000 rpm for $30 \mathrm{~min}$. The obtained P-SLNs were dried at desiccator and store in a cool and dry place. Similarly, placebo SLN was formulated in the same way without incorporation of papain enzyme (Table 1) $[19,20]$.

\section{Particle size and zeta potential analysis}

The mean size and polydispersity index (PDI) of the size distribution for each formula were determined by photon correlation spectroscopy using Zetasizer Nano ZS (Malvern Instruments Ltd., United Kingdom). The SLNs dispersions were diluted in the ratio of 1:1000 with distilled water. The analysis was performed at $25^{\circ} \mathrm{C}$ with an angle of detection of $90^{\circ}$. Each reported value is the average of three measurements. The PDI measures the size distribution of the nanoparticles population [18].

\section{Morphological study}

The morphological characters of the prepared P-SLNs were analyzed under the field emission scanning electron microscope (JEOL JSM6701F JEOL, Japan) (Singh S 2010). The SLNs were mounted on carbon tape and sputter-coated using a thin gold palladium layer under an argon atmosphere using a gold sputter module in a high-vacuum evaporator (JFC-1100 fine coat ion sputter; Jeol, Tokyo, Japan). The coated samples were then scanned, and photomicrographs were taken at an acceleration voltage of $20 \mathrm{Kv}$ [18].

\section{X-ray diffractometry (XRD) studies}

Powder XRD analysis was carried out using Rigaku Ultima IV XRD. The samples were scanned over a $2 \theta$ range of $5^{\circ}-50^{\circ}$ at a step size of $0.045^{\circ}$ and step time of $0.5 \mathrm{~s}$ (Manu Sharma 2011).

\section{Differential scanning calorimetry (DSC) analysis}

The thermal behavior SLN formula was investigated by DSC (Pyris Series Jade DSC). Samples of 2-3 mg were weighed, and a heating rate of $10^{\circ} \mathrm{C} / \mathrm{min}$ was employed in the range of $30^{\circ} \mathrm{C}$ to $380^{\circ} \mathrm{C}$, under a nitrogen purge (40.2 $\mathrm{mL} / \mathrm{min})$ [21].

\section{Entrapment efficiency (EE) and drug loading of P-SLNs}

The percentages of EE (\% EE) of P-SLNs formulations were quantified by spectrophotometrically. The protocol involves the complete extraction of P-SLNs from the nanoparticles through solvents followed by spectrophotometric analysis (Manu Sharma 2011 and Zhiping Zhang). The EE (\%) values were calculated using the following equations;

$$
\% \mathrm{EE}=\frac{\mathrm{W}_{\text {total Papain }}-\mathrm{W}_{\text {free Papain }}}{\mathrm{W}_{\text {total Papain }}} \times 100
$$

\section{In vitro drug release}

In vitro release studies were performed using a dialysis membrane diffusion technique. The dialysis membrane was soaked in a dissolution medium for $12 \mathrm{~h}$ prior usage. The P-SLN dispersion was placed in the dialysis bag; both ends were firmly sealed and immersed into the dialysis medium $(0.1 \mathrm{M} \mathrm{HCl}$ buffer for $2 \mathrm{~h}$ and phosphate buffer pH 6.8 for up to $24 \mathrm{~h}$ ) maintained at $37 \pm 1^{\circ} \mathrm{C}$ and stirred magnetically at $100 \mathrm{rpm}$. At specified time intervals, an aliquot of dialysate was withdrawn, and an equal quantity of fresh dispersion medium was replaced. The aliquot samples were filtered with a $0.1 \mu \mathrm{m}$ filter paper and determined concentration of papain ultraviolet spectroscopy [21].

\section{In vitro cytotoxic potential of P-SLN versus 5-Fu}

Cell culture

Colon adenocarcinoma cell line (human colorectal adenocarcinoma [HCT-15]) was procured from National Centre of Cell Science, Pune, and cultured in RPMI- 1640 medium supplemented with $10 \%$ fetal bovine serum. At confluence, the culture was trypsinized and seeded into a 96well plate in a seeding density of $5 \times 10^{4}$ cells per well. It was incubated overnight to allow attachment.

Drug addition

Eight serial dilutions of pure drug, P-SLN, placebo, and 5-fu (standard anticancer drug; approved against colon cancer by National Cancer Institute, NIH, US Department of Health and Human Services) were 


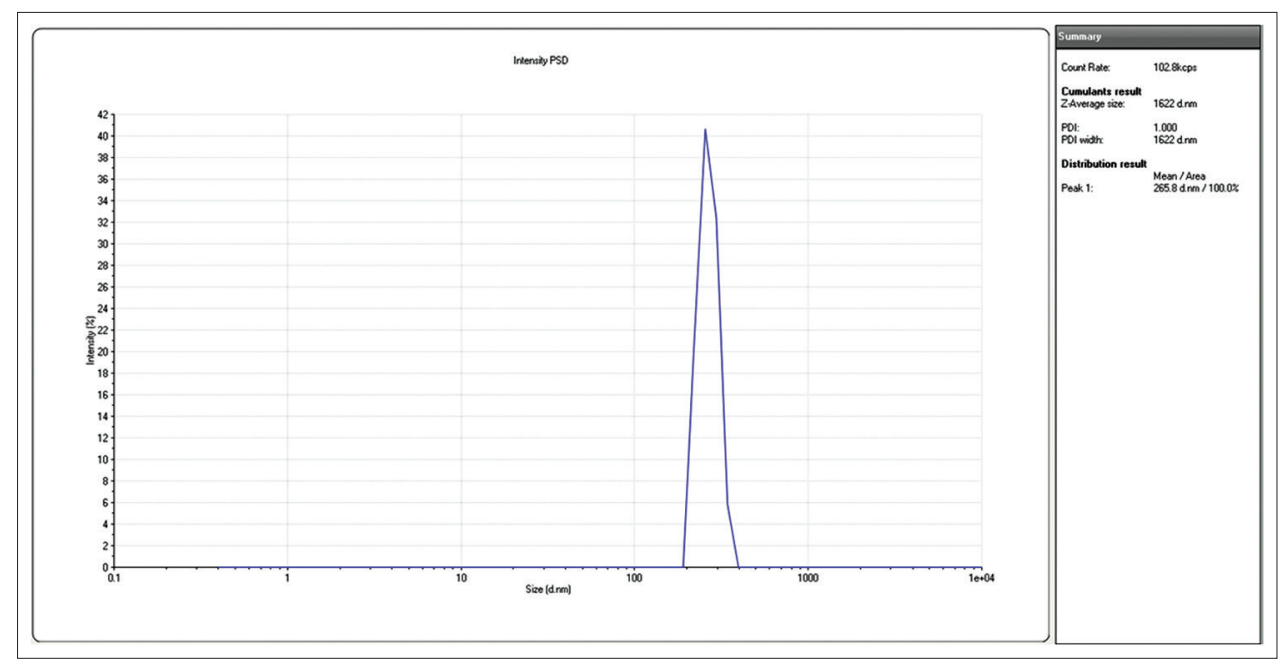

Fig. 2: Particles size of papain-loaded solid lipid nanoparticles

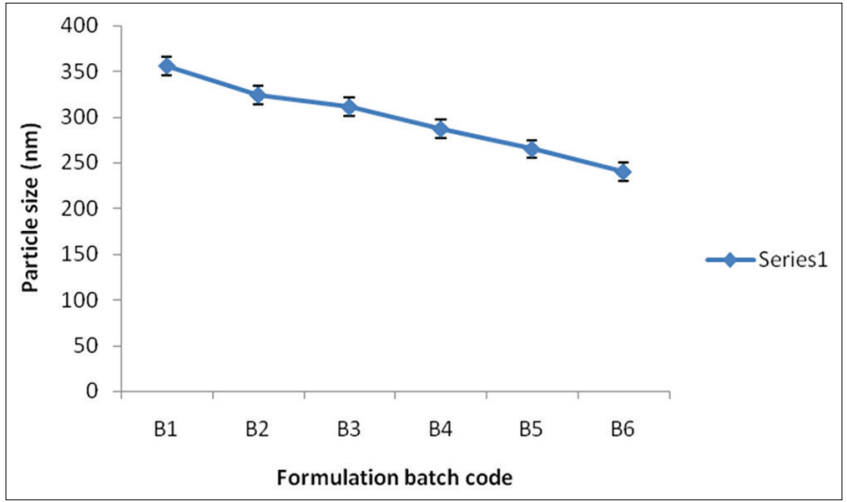

Fig. 3: Mean particle size of papain-loaded solid lipid nanoparticles prepared with the variousdrug: lipid ratio $(1: 1,1: 2$, $1: 3,1: 4$, and 1:5)

made and added to each well in duplicates. The concentrations were $500,250,125,62.5,31.25,15.625,7.812$, and $3.906 \mu \mathrm{g} / \mathrm{ml}$, respectively. Papain was readily soluble in the growth medium. However, as the SLNs were not having an aqueous solubility, they were dispersed in the growth medium and applied to cells. Growth and negative controls were also maintained. The plate was incubated for $24 \mathrm{~h}$ and then processed for determining cell viability.

\section{Determination of cell viability}

At the end of drug exposure period, the growth medium was aspirated from each well, and $50 \mu \mathrm{L}$ of MTT solution $(5 \mathrm{mg} / \mathrm{ml})$ was added to each well. The plate was incubated for $4 \mathrm{~h}$ at $37^{\circ} \mathrm{C}$ in the dark to facilitate the formation of formazan crystals. After incubation $200 \mu \mathrm{L}$ of acidified, DMSO was added to each well to dissolve the formazan crystals to give a purple color. Then, $25 \mu \mathrm{L}$ of glycine buffer was added to all wells and absorbance was measured at $570 \mathrm{~nm}$ by a microplate reader. The $\mathrm{IC}_{50}$ of the samples were calculated from the graph of absorbance along Y-axis against the concentration along X-axis [22].

\section{RESULTS AND DISCUSSION}

Particle size, polydispersity, and zeta potential analysis

Particle size is one of the crucial formulation parameters which will influence the cellular uptake efficiency that governs the adhesive strength between nanoparticles and cellular surface [23]. P-SLN, prepared using cetyl alcohol as the lipid carrier and act as a surfactant, resulted in larger particle size compared to P-SLN prepared using cetyl alcohol with PEG 4000 as a lipid matrix and surfactant, respectively. This could be attributed to the absence of surfactant in this study and cetyl alcohol act as self-surfactant. However, the cetyl alcohol has the ability to produce the solid dispersion with $360 \mathrm{~nm}$ at $1: 1$ druglipid ratio and the particle size was gradually decrease to $265 \mathrm{~nm}$ by increasing the drug-lipid ratio from 1:1 to 1:5. Particle size mainly relies on the carrier material and surfactant concentration. Since cetyl alcohol acts as carrier and surfactant, the particle size was decreased while increasing concentration of cetyl alcohol (Figs. 1-3).

PDI is an assessment of particle whether homogeneous or heterogeneous distribution. The nearer the value of PDI to zero, it indicates that the particles are higher homogenous and the PDI value near to 1 , the particles are non-homogenous nature [19]. The PDI value of P-SLN in batch 1 to batch 4 was recorded $\geq 1$, whereas in batch 5 the PDI was about 1 . Since all the formulation batches have been prepared without surfactant, the PDI value not nearer to 0 .

Zeta potential analysis is a technique for investigating the surface charge of micro/nanoparticles in solution (colloids). It is an essential tool in the prediction of the state of nanoparticle surface and forecasting the storage stability of the nanoparticle. The zeta potential value of PSLN (Batch 5) was recorded $-26.5 \mathrm{mV}$; it shows that the nanodispersion of papain was stable (Fig. 4).

\section{Morphological character}

Nanoparticle morphology is a one of the vital parameters which will determine the cellular uptake [24]. Formulation of nanoparticles with a precise shape such as spherical, cubic, and rod will influence cellular uptake. Particularly spherical shape has the highest cellular uptake than cubic and rod-shaped nanoparticles [25]. Therefore, the morphology of nanoparticles is prominent to achieve greater pharmacokinetics profile of bioactive [26].

The morphological character of PSLN was investigated using scanning electron microscopy, and results showed that the particles were spherical in shape with rough texture (Fig. 5). This might be attributed that the cetyl alcohol acts as a carrier matrix as well it has the potential to reduce the surface tension to form the spherical colloids.

EE

EE is an important parameter which will persuade the in vitro release character for particulate drug delivery systems [19]. To achieve optimal encapsulation efficiency, numerous factors were influenced, including the type and concentration of the lipid, surfactant, and stirring speed [27]. 


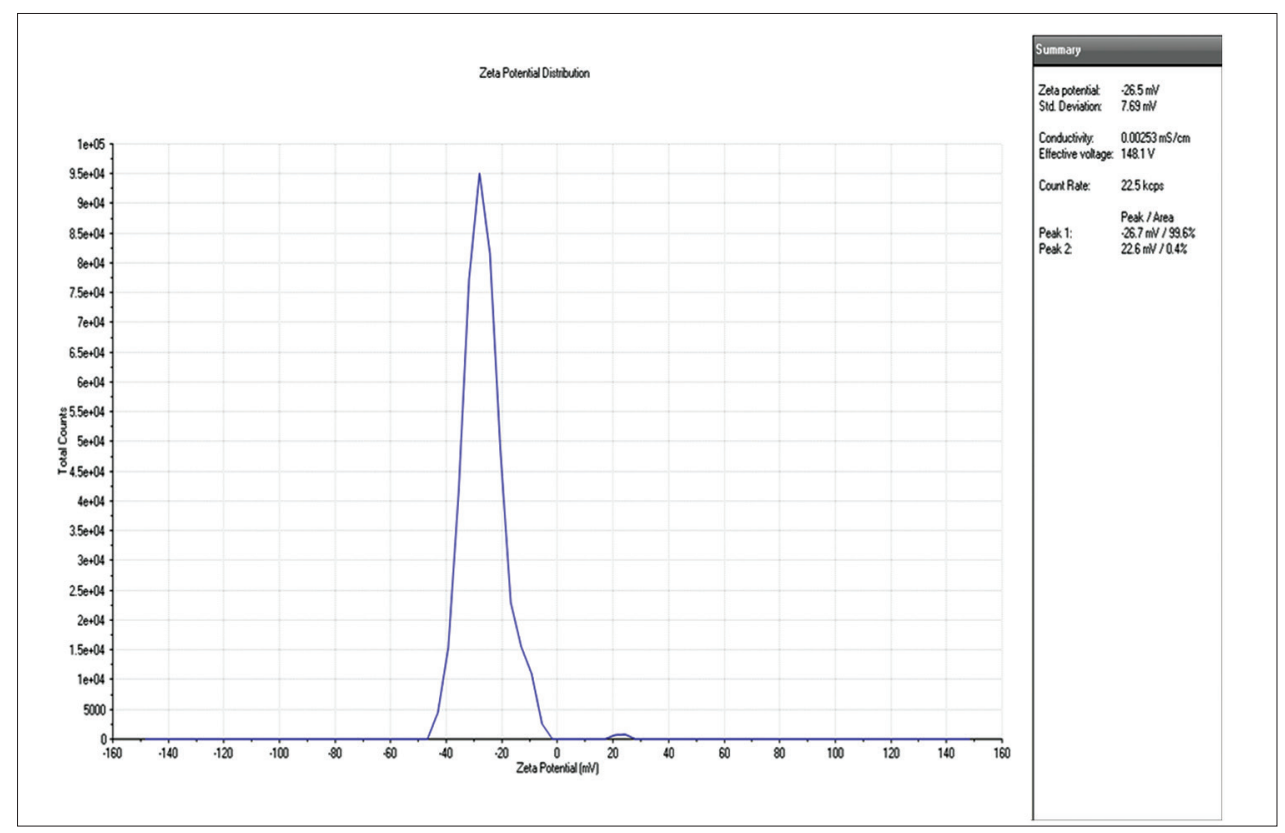

Fig. 4: Zeta potential value papain-loaded solid nanoparticles

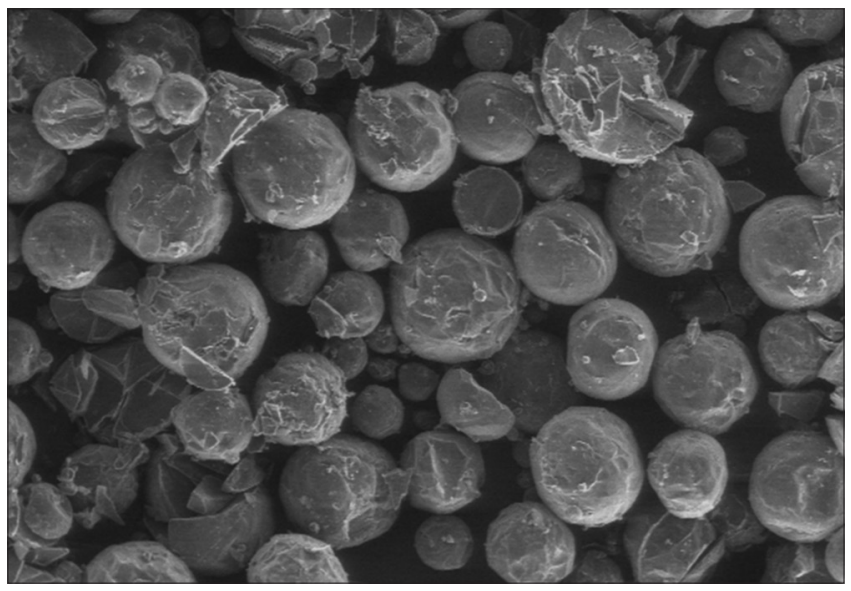

Fig. 5: Scanning electron microscopic image of papain-loaded solid lipid nanoparticles

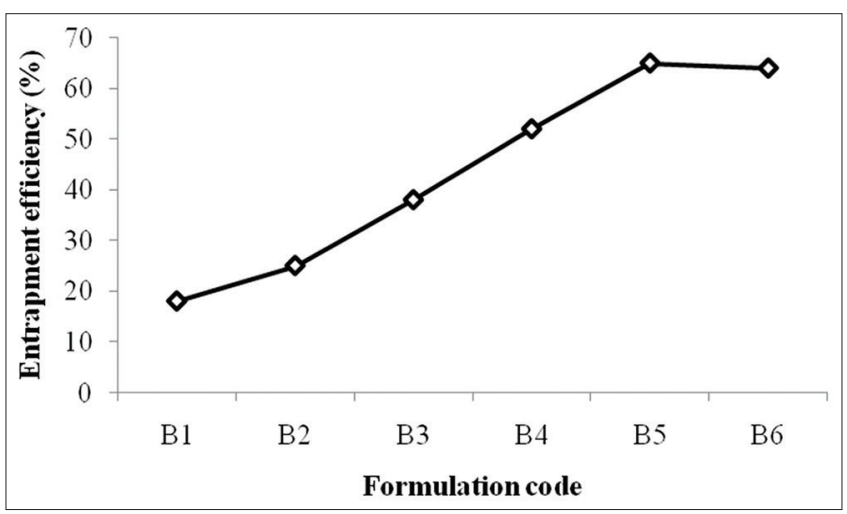

Fig. 6: Entrapment efficiency of papain-loaded solid lipid nanoparticles

Percentages of EE of the P-SLNs are presented in Fig. 6. The \% EE of the P-SLN was obtained in the range of 18.14-65.14\%. The lowest EE was recorded $18 \%$ with $1: 1$ ratio of drug and lipid and the value was gradually increased to $25 \%$ with $1: 2$ ratio of the drug. The maximum amount of EE was recorded 65\% with 1:5 ratios of drug:lipid and $500 \mathrm{rpm}$ as a homogenization speed. Once the homogenization speed increased to $750 \mathrm{rpm}$ with the same drug-lipid ratio (1:5), the EE was slightly decreased to $62 \%$. It indicated that the carrier material and homogenization speed have the capacity to influence the encapsulation efficiency of papain-loaded lipid nanoparticles. It may prove that this cetyl alcohol not only acting as a carrier and but also it acts as a surfactant to influence the drug entrapment into lipid nanoparticles system.

\section{XRD and DSC analysis}

XRD and DSC analysis was performed to understand the physicochemical changes of P-SLN. XRD analysis of papain revealed that the peaks in the $2 \theta$ range of 20-40 inferring its crystalline nature (Fig. 7). However, in the P-SLN, no such crystalline peaks were observed. Thus, it confirms the amorphous of papain in the SLN. As seen in the XRD analysis, alteration of crystallinity is essential because this was strongly connected with drug entrapment and drug release rate.

According to DSC analysis, papain exhibited a characteristic endothermic peak at $180^{\circ} \mathrm{C}, 220^{\circ} \mathrm{C}$, and $250^{\circ} \mathrm{C}$ due to its crystalline nature, while the P-SLN exhibited no peak in this region (Fig. 8). These results suggest that crystalline form of papain was converted into amorphous nature, which has more solubility and stability. Further, the absence of papain characteristic peak in SLN represents that the drug was incorporated into the lipid carrier [28].

\section{In vitro drug release studies}

The in vitro drug release profile of papain from various SLN formulations is shown in Fig. 9. The in vitro release of P-SLN was found to be in the range of $68.92-84.32 \%$ at the end of $12 \mathrm{~h}$. Formulations containing cetyl alcohol as a lipid carrier (1:1 drug:lipid) exhibited higher drug release than the formulations containing 1:5 drug:lipid ratio, which showed a more sustained release. The cumulative percentage of drug release of P-SLN was on the basis of the papain has coated with the amount of lipid carrier. Thus, it could be well concluded that the amount of papain released was sustain manner when SLN was prepared by using 1:5 ratio of drug and lipid.

\section{Cell viability studies on HCT-15 cell line}

An ideal cytotoxic agent should have IC I0 $_{50}$ value as less as possible [29]. In recent decades, chemotherapy is used as an essential element of a multimodal approach to the treatment of many types of cancers, 


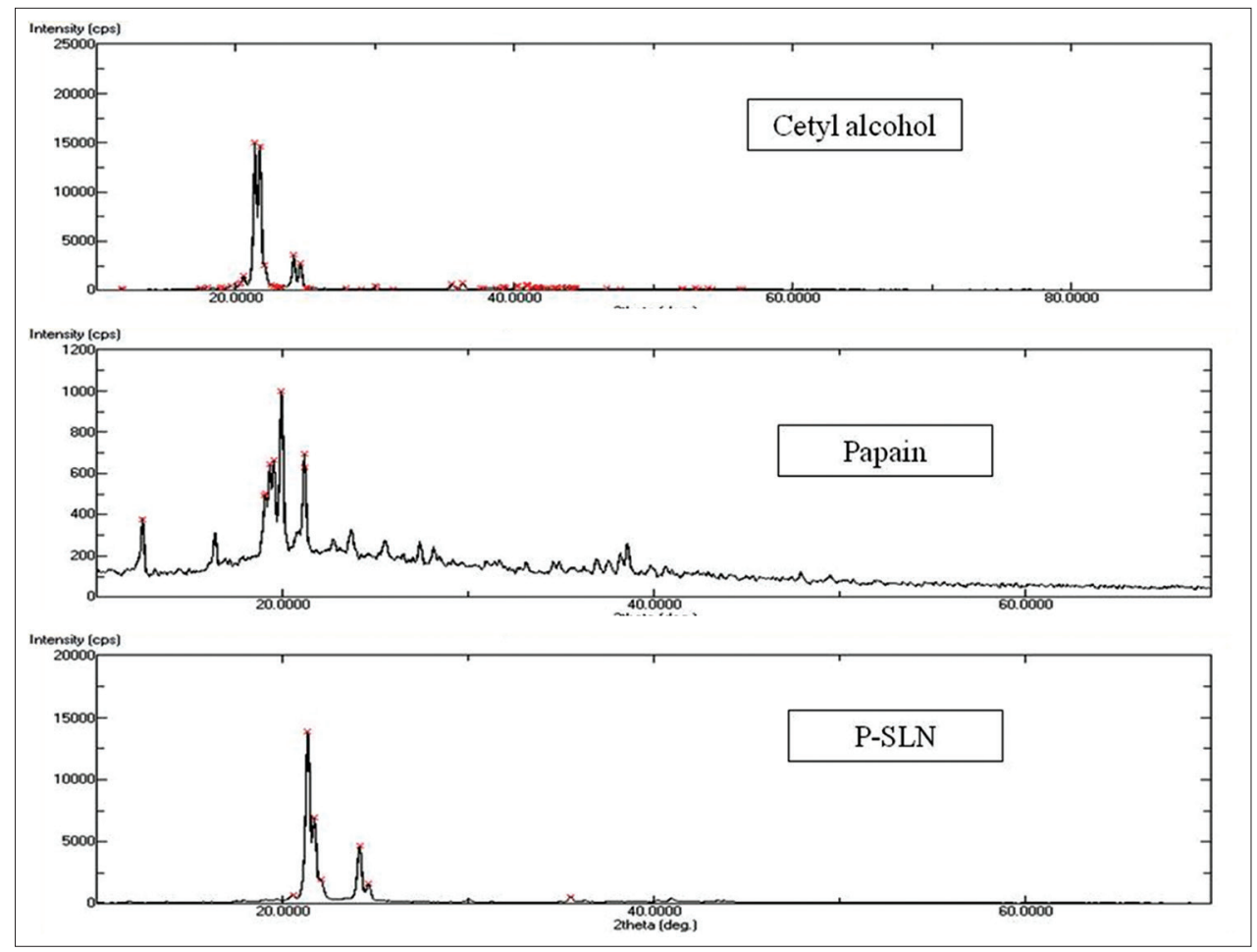

Fig. 7: X-ray diffractogram of cetyl alcohol, papain, and papain-loaded solid lipid nanoparticles

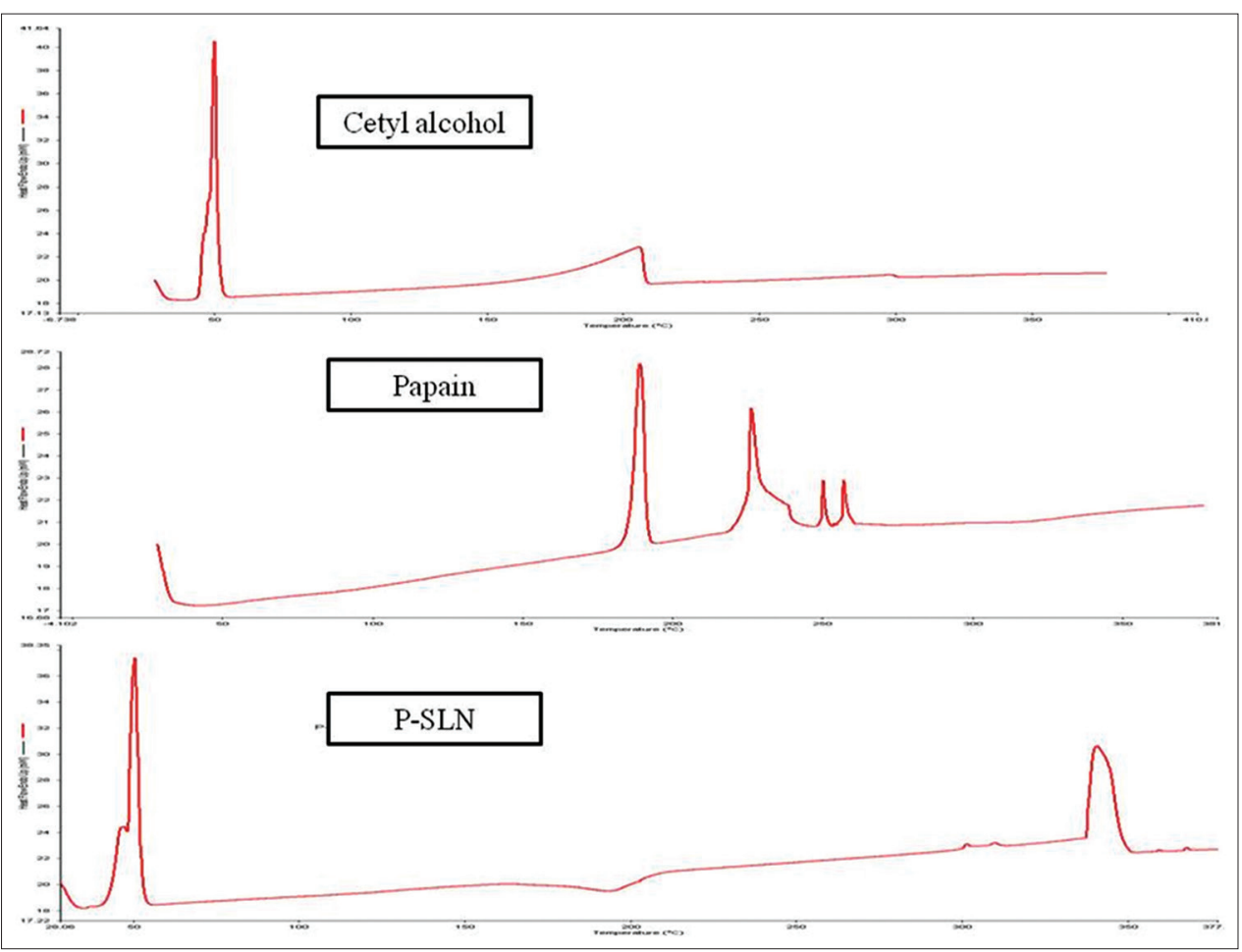

Fig. 8: Differential scanning calorimetric analysis of cetyl alcohol, papain, and papain-loaded solid lipid nanoparticles

including locally advanced stages of esophageal, lung, colorectal, head and neck cancer, and soft tissue sarcomas [30]. Nanoparticulate drug delivery possesses the remarkable potential for overcoming most of the problems that conventional delivery face in the treatment, diagnosis, and detection of cancer [31]. To overcome the conventional delivery drawbacks, the papain has loaded into solid lipid nanoparticles to achieve better anticancer potential than pure papain. Different drugs have different levels of cytotoxicity toward different cells. This drug may not be an appropriate cytotoxic agent against colorectal cell lines. However, the same may be effective against some different cell line(s). The cytotoxicity effect of pure papain enzyme was not that convincing $\left(\mathrm{IC}_{50}=477 \mu \mathrm{g} / \mathrm{mL}\right.$ against $13 \mu \mathrm{g} / \mathrm{mL}$ of $\left.5-\mathrm{Fu}\right)$. P-SLNs have shown enhanced cytotoxicity as compared to the pure papain $\left(\mathrm{IC}_{50}=50 \mu \mathrm{g} / \mathrm{mL}\right)$. The enhanced cytotoxic efficacy of P-SLN has achieved because the lipidic carrier has the tendency to facilitate to easy uptake by cancerous cells. This proved that papain-loaded 


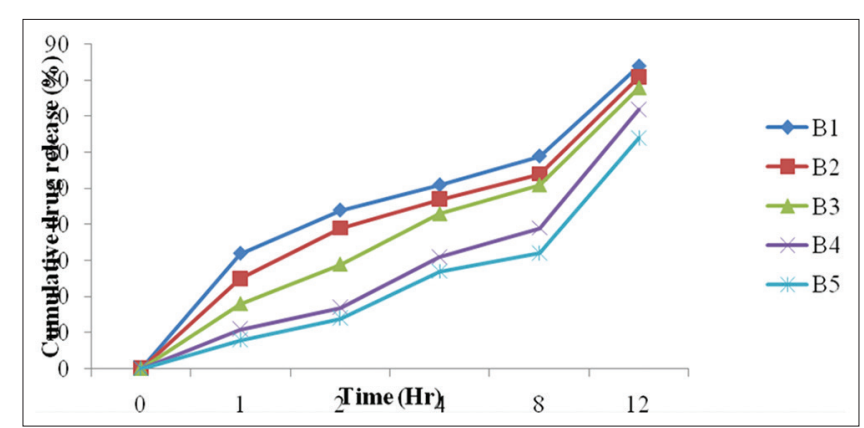

Fig. 9: In vitro cumulative drug release profile of papain-loaded solid lipid nanoparticles

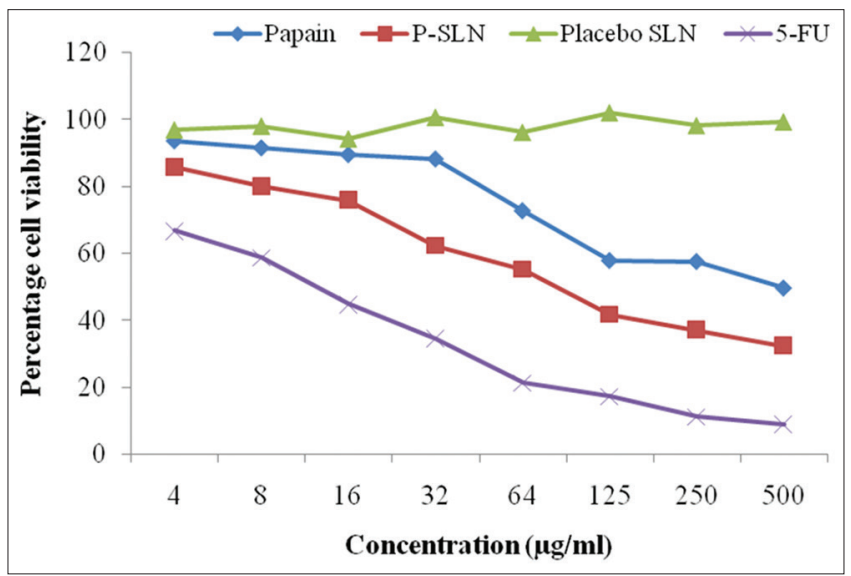

Fig. 10: Percentage cell viability of human colorectal adenocarcinoma cell line
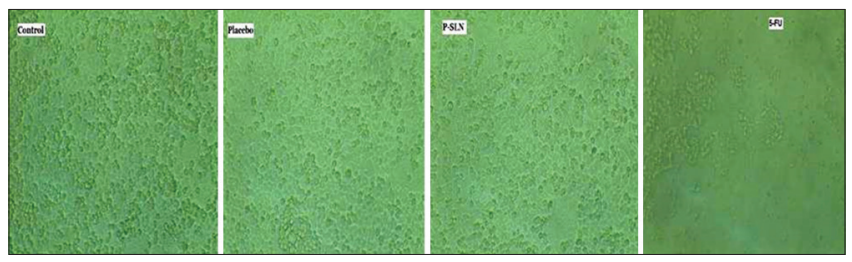

Fig. 11: Cell viability study of papain and papain-loaded solid lipid nanoparticles compared with 5-fluorouracil on human adenocarcinoma human colorectal adenocarcinoma cell line

nanoparticles have better cytotoxic potential than pure papain. The placebo, as expectedly, did not show any cytotoxic activity. It evidenced that the SLN carrier materials did not responsible for the cytotoxic to the cancer cells (Figs. 10 and 11).

\section{CONCLUSION}

Papain is a powerful proteolytic enzyme (Carica papaya L.) which is obtained from the latex of greenish papaya fruits. Papain has the ability to break down organic molecules made of amino acids, known as polypeptides and hence plays a crucial role in various biological processes in physiological and pathological states, pharmaceutical drug designs. An aqueous extract of $C$. papaya was also reported for its effect on the growth of various tumor cell lines and human lymphocytes and have shown positive significant results. However, papain has not explored as an anticancer compound as compared with $C$. papaya crude extracts and also reported with poor target specificity, deprived bioavailability due to gastric degradation. To overcome these setbacks, P-SLN and investigated the in vitro drug release and in vitro cell viability against HCT 15 cell line. The P-SLN has revealed that it produced sustained drug release and least cell viability as compared with pure papain enzyme. With this evidence, further investigation needs to be executed including cell death (HCT 15) mechanism and preclinical study to make available this P-SLN as adjuvant therapy or chemotherapy for the treatment of colorectal cancer.

\section{AUTHOR'S CONTRIBUTION}

Author Suriyakala Perumal Chandran has contributed for planning and execution of research and experimentation. Author Kannikaparameswari Nachimuthu who has contributed for data interpretation, manuscript construction, and editing.

\section{CONFLICTS OF INTEREST}

The authors declared that there are no conflicts of interest between as from this research and publication.

\section{REFERENCES}

1. Cisterna BA, Kamaly N, Choi WI, Tavakkoli A, Farokhzad OC, Vilos C, et al. Targeted nanoparticles for colorectal cancer. Nanomedicine (Lond) 2016;11:2443-56

2. Goldberg RM, Rothenberg ML, Van Cutsem E, Benson $A B 3^{\text {rd }}$, Blanke CD, Diasio RB, et al. The continuum of care: A paradigm for the management of metastatic colorectal cancer. Oncologist 2007;12:38-50.

3. Fornarini G, Guglielmi A, Sobrero A. The handling of metastatic colorectal cancer. Ann Oncol 2005;16 Supplement 2:141-3.

4. Dallas NA, Xia L, Fan F, Gray MJ, Gaur P, van Buren G $2^{\text {nd }}$, et al. Chemoresistant colorectal cancer cells, the cancer stem cell phenotype, and increased sensitivity to insulin-like growth factor-I receptor inhibition. Cancer Res 2009;69:1951-7.

5. Lipinski B. Iron-induced parafibrin formation in tumors fosters immune evasion. Oncoimmunology 2014;3:e28539.

6. Keith IB, Barry BD, Nicholas G, Aristo V. The Immune system in cancer. Integrative Cancer Ther 2002;1:294-316.

7. Vicente LC, Aires-Barros R, Empis JM. Stability and proteolytic activity of papain in reverse micellar and aqueous media: A kinetic and spectroscopic study. J Chem Technol Biotechnol 1994;60:291-7.

8. Mukherjee S, Ray S, Thakur RS. Solid lipid nanoparticles: A modern formulation approach in drug delivery system. Indian J Pharm Sci 2009; $71: 349-58$.

9. Gamal AS. Ciprofloxacin controlled-solid lipid nanoparticles: Characterization, in vitro release, and antibacterial activity assessment. Biomed Res Int 2017;2120734:9.

10. Pooja D, Tunki L, Kulhari H, Reddy BB, Sistla R. Optimization of solid lipid nanoparticles prepared by a single emulsification-solvent evaporation method. Data Brief 2016;6:15-9.

11. Freitas C, Müller RH. Correlation between long-term stability of solid lipid nanoparticles (SLN) and crystallinity of the lipid phase. Eur J Pharm Biopharm 1999;47:125-32.

12. Liu T, Choi H, Zhou R, Chen IW. Quantitative evaluation of the reticuloendothelial system function with dynamic MRI. PLoS One 2014;9:e103576.

13. Cavalli R, Gasco MR, Chetoni P, Burgalassi S, Saettone MF. Solid lipid nanoparticles (SLN) as ocular delivery system for tobramycin. Int J Pharm 2002;238:241-5.

14. Wissing SA, Kayser O, Müller RH. Solid lipid nanoparticles for parenteral drug delivery. Adv Drug Deliv Rev 2004;56:1257-72.

15. Padhye SG, Nagarsenker MS. Simvastatin solid lipid nanoparticles for oral delivery: Formulation development and in vivo evaluation. Indian J Pharm Sci 2013;75:591-8.

16. Tupal A, Sabzichi M, Ramezani F, Kouhsoltani M, Hamishehkar H. Dermal delivery of doxorubicin-loaded solid lipid nanoparticles for the treatment of skin cancer. J Microencapsul 2016;33:372-80.

17. Attama AA, Umeyor CE. The use of solid lipid nanoparticles for sustained drug release. Ther Deliv 2015;6:669-84

18. Thiresen G, Yahya EC, Pradeep K, Lisa CT, Girish M, Dinesh N, et al. A novel melt-dispersion technique for simplistic preparation of chlorpromazine-loaded polycaprolactone nanocapsules. Polymers 2015:7:1145-76.

19. Uprit S, Kumar Sahu R, Roy A, Pare A. Preparation and characterization of minoxidil loaded nanostructured lipid carrier gel for effective treatment of alopecia. Saudi Pharm J 2013;21:379-85.

20. Satheesh BN, Prabakaran L. formulation of ofloxacin loaded lipospheres 
with improved oral bioavailability. Pharm Nanotechnol 2013;1:306-15.

21. Sharma M, Sharma V, Panda AK, Majumdar DK. Development of enteric submicron particle formulation of papain for oral delivery. Int $\mathbf{J}$ Nanomedicine 2011;6:2097-111

22. Subramanian AP, Jaganathan SK, Mandal M, Supriyanto E, Muhamad II. Gallic acid induced apoptotic events in HCT-15 colon cancer cells. World J Gastroenterol 2016;22:3952-61.

23. Elias DR, Poloukhtine A, Popik V, Tsourkas A. Effect of ligand density, receptor density, and nanoparticle size on cell targeting. Nanomedicine 2013;9:194-201.

24. Albanese A, Tang PS, Chan WC. The effect of nanoparticle size, shape, and surface chemistry on biological systems. Annu Rev Biomed Eng 2012;14:1-6

25. Karagoz B. Polymerization-induced self assembly (PISA)-control over the morphology of nanoparticles for drug delivery applications. Polym Chem 2014;5:350-5.
26. Nazanin H, Samantha G, Hongbin H, Gang B. The effect of nanoparticle size on in vivo pharmacokinetics and cellular interaction. Nanomedicine 2016;11:673-92

27. Ekambaram P, Abdul HS. Formulation and evaluation of solid lipid nanoparticles of ramipril. J Young Pharm 2011;3:216-20.

28. Dudhipala N, Janga KY. Lipid nanoparticles of zaleplon for improved oral delivery by box-behnken design: Optimization, in vitro and in vivo evaluation. Drug Dev Ind Pharm 2017;43:1205-14

29. Heidi AD. Efficacy of cancer drugs: Looking beyond the $\mathrm{IC}_{50}$. Chem Res Toxicol 2013; 26:1776-77.

30. Minakshi G, Jyoti D, Rakesh KM, Harish D. Therapies in cancer treatment: An overview. Int J Pharm Pharm Sci 2015;7:1-9.

31. Ksy H, Abhay R, Praveen S, Swati U, Hemanth K. Cancer nanotechnology: Nanoparticulate drug delivery for the treatment of cancer. Int J Pharm Pharm Sci 2015;7:40-6 